\title{
Correlation length around Mars: A statistical study with MEX and MAVEN observations
}

\author{
Adriane Marques de Souza Franco ${ }^{1 *}$, Markus Fränz ${ }^{2}$, Ezequiel Echer ${ }^{1}$, and Mauricio José Alves Bolzan ${ }^{3}$ \\ ${ }^{1}$ National Institute for Space Research (INPE), Sao Jose dos Campos, Brazil; \\ ${ }^{2}$ Max Planck Institute for Solar System Research, Goettingen, Germany; \\ ${ }^{3}$ Federal University of Jataí, Jataí, Brazil
}

\begin{abstract}
Correlation lengths of ultra-low-frequency (ULF) waves around Mars were computed for the first time, using data from MEX (electron density from 2004 to 2015) and MAVEN (electron density and magnetic field from 2014 to 2016). Analysis of the MEX data found that, for the frequency range 8 to $50 \mathrm{mHz}$, correlation length in electron density varied between 13 and 17 seconds (temporal scale) and between $5.5 \times 10^{3} \mathrm{~km}$ and $6.8 \times 10^{3} \mathrm{~km}$ (spatial scale). For the MAVEN time interval, correlation length was found to vary between 11 and 16 seconds (temporal scale) and $2 \times 10^{3}-4.5 \times 10^{3} \mathrm{~km}$ in spatial scale. In the magnetic field data, correlation lengths are observed to be between 8-15 seconds (temporal scale) and between $1 \times 10^{3}$ and $5 \times 10^{3} \mathrm{~km}$ (spatial scale) over the same frequency range. We observe that the cross sections of the plasma regions at the dayside of Mars are smaller than these correlation lengths in these regions in both analyses, where the correlation length derived from the MEX electron density data was between 5 and 25 times the size of the magnetosheath and the magnetic pile-up region (MPR), respectively. For MAVEN these ratios are about 4 (magnetosheath) and 11 (MPR) in electron density and between 1.5 and 5.5 for magnetic field data, respectively. These results indicate that waves at the magnetosheath/MPR can be related to oscillations in the upper ionosphere. In a local region, wave trains may cause resonance effects at the planetary ionopause, which consequently contributes to the enhanced ion escape from the atmosphere.
\end{abstract}

Keywords: Mars induced magnetosphere; ULF waves; correlation length

Citation: Franco, A. M. S., Fränz, M., Echer, E., and Bolzan, M. J. A. (2019). Correlation length around Mars: A statistical study with MEX and MAVEN observations. Earth Planet. Phys., 3(6), 560-569. http://doi.org/10.26464/epp2019051

\section{Introduction}

Mars has an induced magnetosphere caused by induction of electric fields in the upper ionosphere by the magnetized solar wind (Kivelso and Bagenal, 2007). Since the ionosphere of Mars forms a conductive layer, the solar wind magnetic field cannot easily penetrate and the deflected fields produce induced currents in the upper ionosphere. As a consequence, the magnetic field piles up in front of the planet, which causes the incident solar wind plasma to slow down and be deflected around the planet, forming a magnetosheath region similar to the magnetosphere that is created by an intrinsic magnetic field (Cloutier and Daniell, 1973, 1979; Podgorny et al., 1980).

In the Mars orbit, the passage of interplanetary shock waves, ICMEs (Interplanetary Coronal Mass Ejections), and CIRs (Corotating Interaction Regions) perturb the solar wind; consequently, ion and energetic electron flows associated with these shocks can reach the planet. As Mars does not have an intrinsic magnetic field, the planet cannot totally deflect the solar wind particles, especially those of high energy; some of them can penetrate into

Correspondence to: A. M. S. Franco, adrianemarquesds@gmail.com Received 02 MAR 2019; Accepted 13 AUG 2019.

Accepted article online 27 SEP 2019.

(C) 2019 by Earth and Planetary Physics. the atmosphere of Mars (Luhmann et al., 1992, 2004). Another factor that can transfer energy from solar wind to the inner magnetosphere is propagation of Ultra-Low Frequency (ULF) waves (Kivelson, 1995). Mainly generated at the bow shock upstream region by heavy ions, reflecting protons and electrons at this boundary, ULF waves are observed in the foreshock due to energized particles of the solar wind, which get their energy from multiple crossings of the bow shock and escape to the upstream region, creating instabilities in the foreshock's plasma. Those waves are also generated due to magnetosheath ions that escape across the bow shock and move in the anti-solar region (Russell et al., 1990; Delva and Dubinin, 1998; Volwerk et al., 2008). In the upstream region of Mars, ULF waves show a strong dependence on the interplanetary magnetic field (IMF) orientation. It was observed that when the IMF is more radial, the observed waves are more intense (Halekas et al., 2017). The radial geometry facilitates upstream movement of the ions when reflected from the bow shock, which contributes to the formation of upstream instabilities.

It was observed earlier that high ULF wave activity (sufficient to reach the ionospheric region and heat the plasma) is correlated with greater ionospheric ion acceleration (Lundin et al., 2011). This process can provide enough energy to planetary ions for them to escape from the planet. Consequently, ULF waves play an import- 
ant role in the atmospheric loss on Mars. Winningham et al. (2006) observed that ULF waves in the magnetosheath region sometimes present frequencies similar to the gyrofrequency of oxygen in the region, and these waves can be connected to waves in the ionosphere. Recent studies by Collinson et al. (2018) using MAVEN (Mars Atmosphere and Volatile EvolutioN) and MEX (Mars EXpress) data have shown that compressive ULF waves in the solar wind can drive magnetosonic ULF waves in the ionosphere of Mars and that these pulsations can heat and energize the plasma from the ionosphere.

In order to estimate the interaction region size of ULF waves in different propagation directions in the terrestrial foreshock, Archer et al. (2005) used data from the four Cluster spacecraft to compute the correlation length of ULF waves observed in the magnetic field. These authors determined that the correlation length is typically 8-18 Earth's radius in the perpendicular direction to the wave vector, and 1-3 Earth's radius along the wave vector. This study is useful for understanding the wave-particle interactions.

In order to determine whether ULF waves, when converted to compressive pulses, are capable of causing resonance effects at the ionopause of Mars, in this work we compute correlation lengths $(C L)$ around Mars for the observation intervals of MEX (2004-2015) and MAVEN (2014-2016) and compare the values of $C L$ in each plasma region of the dayside magnetosphere statistically with the size of the specific region.

\section{Materials and Methods}

The general definition of the correlation length is a characteristic scale over which fluctuations in a variable are correlated (Fisk and Sari, 1973). In other words, the correlation length is the distance from a given point to the most distant point beyond which there is no further correlation of a physical property associated with the given point (Mela and Louie, 2001; Wicks et al., 2010). The correlation length has many applications in different areas, such as in the study of structures in the solar wind (Fisk and Sari, 1973; Wicks et al., 2010), molecular dynamics simulation (Morales and Nuevo, 1993), scattering of cosmic rays (Parhi et al., 2002), and turbulent boundary layer flight data (Palumbo, 2012), among others.

Since our study is in the ULF range, before computing the correlation length we apply a Fourier transform to remove high frequencies present on the data. Because the highest power in foreshock ULF waves is typically between 8 and $50 \mathrm{mHz}$ (Fränz et al., 2017), we define this to be the relevant frequency domain. We then use the inverse Fourier transform to transform the data back to the time domain. To compute the correlation length, we calculate the autocorrelation function $(A C)$, lagged by a time between 0 and 60 $\mathrm{s}$ and sliding a window of $120 \mathrm{~s}$ width across the data. This restricts the possible detection of correlation lengths to the range 8-60 s.

The $A C$ curve can be represented by an exponential of the lag $(L)$ by the biggest lag $(C L)$ :

$$
A C(L)=\mathrm{e}^{(-L / C L)}
$$

where $C L$ is the correlation length.
To obtain the correlation length, an exponential fit must be employed to the auto-correlation amplitude curve:

$$
\ln A C(L)=\frac{L}{C L}
$$

Then,

$$
C L=\frac{L}{\ln A C(L)}
$$

After that, a linear regression $\left(Y=A_{0}+A_{1} X+\ldots\right)$ has been employed to find $C L$ from Equation (2).

The correlation length can then be found as:

$$
C L=\left|\frac{1}{A_{1}}\right| \text {. }
$$

Here the $C L$ are given in a temporal scale; to obtain the correlation length in a spatial dimension $\left(C L_{S}\right)$, the temporal correlation length $\left(C L_{T}\right)$ must be multiplied by the plasma velocity $\left(V_{S W}\right)$, as Equation (4) shows.

$$
C L_{S}=C L_{T} \cdot V_{S W}
$$

In our analysis we used the ASPERA-3/IMA (MEX) and SWIA (MAVEN) velocity interpolated to the same timetags as those of the electron or magnetic field data.

\section{Data}

In this paper, our data come from the MEX (covering the interval 2004-2015) and MAVEN (2014-2016 spacecraft — in particular, from the ELS/ASPERA-3 (Electron Spectrometer/Analyzer of Space Plasma and Energetic Atoms Experiment) aboard MEX (Barabash et al., 2004) and from MAVEN's Solar Wind Electron Analyzer (SWEA) (Mitchell et al., 2016). Electron densities have been calculated according to the methods described in Fränz et al. (2006). As MEX does not have a magnetometer, the magnetic field correlation length has been calculated using only data from the magnetometer on board MAVEN (Connerney et al., 2015a, b). All data are downsampled to $4 \mathrm{~s}$ time resolution.

\section{Results}

The correlation length analysis was developed by building maps that present statistical probability, in order to observe a certain correlation length in a spatial bin around the planet. Each bin has a dimension of $0.1 \times 0.1$ Mars radii in cylindrical MSO (Mars Solar Orbit) coordinates. The $x$-axis represents the Mars-Sun line and is centered in Mars; it varies from $-4 R_{\mathrm{M}}$ to $3 R_{\mathrm{M}}$. The $y$-axis represents the $y$ direction and varies from 0 to $4.5 R_{\mathrm{M}}$. All figures indicate the main localization of the boundaries (Bow shock and magnetic pile up boundary - MPB) of the Mars magnetosphere found by Han et al. (2014).

When the dynamic pressure of the solar wind is high, it increases wave production in the magnetosheath (Lundin et al., 2008). In order to determine whether the size of the structures around the planet change with the solar wind pressure, we performed a study of the correlation length during periods of low (between $0 \mathrm{nPa}$ and $1 \mathrm{nPa}$ ) and high (between $1 \mathrm{nPa}$ and $100 \mathrm{nPa}$ ) solar wind pressure values for the 2004 to 2012 time interval. It is important to mention that this analysis is based on 86 intervals selected for 
high pressure and 650 selected for low pressure. That difference occurs because IMA data are used for SWP analysis and those data have gaps. The solar wind pressure was determined from daily and $12 \mathrm{~h}$ averages of the upstream MEX/IMA ion observations. From this dataset we chose time intervals of low and high solar wind pressure.

Before presenting the statistical analyses, we provide an example of our correlation length analysis applied to the electron density (ELS/ASPERA-3/MEX) data. This example, from 08:30 UT February
01, 2012 to 10:00 UT, February 01, 2012 in temporal and spatial scale, is presented in Figure 1.

Figure $1 \mathrm{a}$ shows the electron density time series; Figure $1 \mathrm{~b}$, the same time series after Fourier Transform processing; Figure 1c, the correlation length in temporal scale. Figure 1d presents IMA velocity and Figure 1e, the correlation length in spatial scale. In Figure $1 \mathrm{c}$ it is possible to observe that correlation lengths show peaks up to 40 seconds, but lower values are predominant (10-30 seconds). Spatial scale correlation lengths (Figure 1e) are on the order of $1 \times$
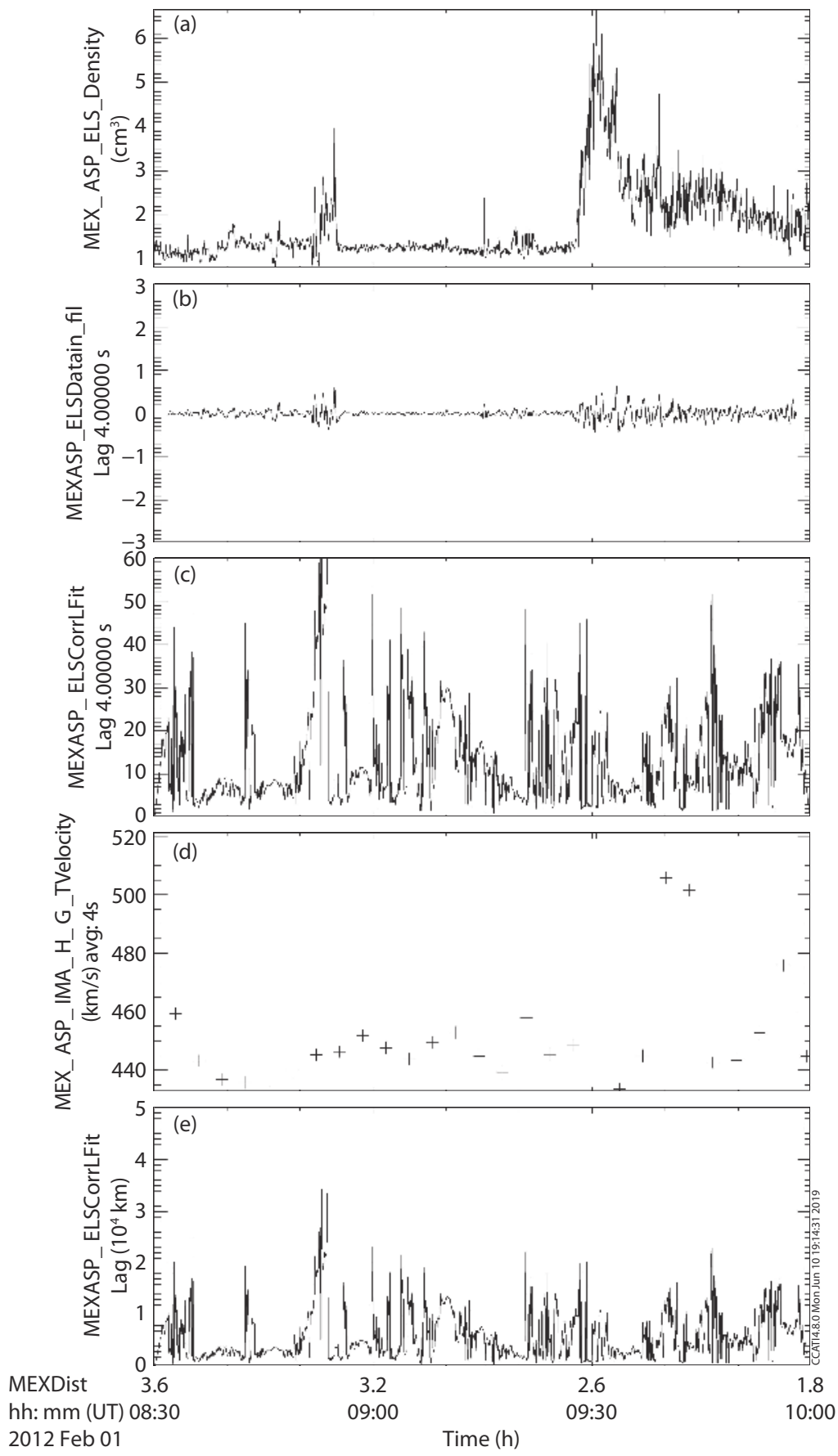

Figure 1. Example of the correlation length analysis applied to MEX electron density data from 08:30 UT February 01, 2012 to 10:00 UT, February 01, 2012. (a) Electron density time series; (b) time series after the Fourier Transform processing; (c) correlation length in temporal scale; (d) IMA velocity and (e) correlation length in spatial scale. 
$10^{4} \mathrm{~km}$. The statistical analyses of correlation length are presented for temporal and spatial scales; results will be shown in the next subsection, divided by results for each spacecraft (MEX and MAVEN).

\subsection{MEX Results}

As mentioned before, MEX does not have a magnetometer. Thus, the correlation lengths for its data were calculated only for electron density data.

\subsubsection{Temporal scale}

The $C L_{T}$ calculation results are presented as maps showing their distribution in relation to the planet and (nominal) MPB and bow shock positions. In temporal scale, $C L_{T} S$ are represented in color scale, which varies between 8 and 20 seconds. Dashed red bins represent regions where $C L_{T} S$ are higher than the maximum value of the scale, black dashed bins where they are lower than the minimum, and white bins for regions where no data were available. In our analyses we have used the highest value of $C L_{T}$ in each boundary/region as reference.

Figure 2a shows $C L_{T} S$ for the entire interval covered by MEX data. Figures $2 \mathrm{~b}$ and $2 \mathrm{c}$ present, respectively, $C L_{T} s$ during periods of low and high solar wind pressure. In Figure $2 \mathrm{a} C L_{T}$ is high in the magnetotail (varing between 13 and 15 seconds). The most significant outflow of particles is observed in the region of the Mars magnetotail (Lundin et al., 2006). High correlation length values observed in the magnetotail may be related to tail ion beams with energy levels similar to those of solar wind protons (Barabash et al., 2004; Lundin et al., 2006). It is also possible to observe high values of $C L_{T} S$ at the nose of the planet, from the solar wind to lower regions of the magnetosheath, which may indicate wave penetration into the upper ionosphere of Mars. Looking at the Figures $2 b$ and $2 \mathrm{c}$, it is clear that during high solar wind pressure intervals (Figure 2c), larger $C L_{T}$ values can be observed in all regions. But during periods of low solar wind pressure (Figure $2 \mathrm{~b}$ ), high $C L_{T}$ values are observed mainly in the inner regions of the magnetosphere, dayside MPR ( 17-18.5 seconds), and tail (15-18 seconds). The intervals of high pressure correspond to perturbed periods near the Mars environment due to the presence of ICME and CIRS, which can increase correlation length values.

These differences between low and high solar wind pressure may indicate that either the heavy ion exosphere or reflected ions are able to destroy ULF wave trains that are transported by the solar wind during low solar wind pressure intervals. In this analysis one can also notice that the bow shock moves toward the planet during periods of high solar wind pressure (Figure 2c), as compared with periods of low solar wind pressure (Figure $2 b$ ), which agrees with the results of other studies (Trotignon et al., 1993; Schwingeschuh et al., 1992; Ruhunusiri et al., 2015b; Halekas et al., 2017; Ramstad et al., 2017).

Lundin et al. (2008) have shown that the planetary ion escape rate grows with increasing solar wind pressure, and that the solar wind pressure controls the energy and momentum transfer to planetary ions. Further, ULF wave intensity is observed to be directly correlated with solar wind dynamic pressure (Lundin et al., 2011). Thus, high values of correlation length observed in the whole magnetosphere during periods of high solar wind pressure also indicate the importance of these ULF waves for the escape of atmospheric ions from Mars.

\subsubsection{Spatial scale}

In order to obtain the correlation length in spatial scale, correlation length in temporal scale was multiplied by the instantaneous plasma velocity. The maps of the correlation length in spatial scale are presented in Figure 3. The color scale here represents the correlation length in kilometers, with the scale varying from $1 \times 10^{3}$ to $8 \times 10^{3} \mathrm{~km}$. For the whole analyzed interval (Figure 3a), $C L_{s} s$ are lower in terminator, in the inner region of the magnetosphere. At the nose of the planet, $C L_{S}$ s show higher values from the foreshock, throughout the whole magnetosheath region, down to the MPR. In spatial scale, correlation length is clearly larger in the region of the magnetosheath in general, but mainly at the nose of the planet. Espley et al. (2004) have shown that transverse waves are the dominant wave mode at the nightside of the magnetosheath, whereas compression waves dominate at the dayside. They associate these waves to Martian locally picked-up Martian ions (which are relatively cold), that by interaction with solar wind protons produce oscillations in the magnetic field. Ruhunusiri et al. (2015a) observed that the Alfven wave mode is the wave mode dominant in the Martian magnetosheath. These waves can propagate in the magnetosphere and may be converted to compressionable modes which can penetrate through the MPB (Lundin et al., 2011; Ruhunusiri et al., 2015a).

In spatial scale (Figure 3), as was observed in the temporal analysis (Figure 2), during the periods of high pressure (Figure $3 \mathrm{c}$ ), $C L_{s} S$ are larger in all regions (except for the MPR), compared to values observed in periods of low pressure (Figure $3 \mathrm{~b}$ ). It is important to mention that, the spatial scale correlation length is computed using the proton plasma velocity; this can affect correlation length values during periods of high pressure intervals, since the fluctuations in this parameter increase. But it is also important to point out the fact that an increase in the $C L_{s}$ during high pressure intervals was observed also in the temporal analysis, which is computed without using the plasma velocity.

\subsection{MAVEN Results}

The analyses of the MAVEN data were performed for the interval from November 2014 to December 2016. As MAVEN has a magnetometer onboard, besides the electron density analysis we were able to calculate magnetic field correlation length. The analysis here is presented in temporal and spatial scales for the whole period. Due to MAVEN's shorter temporal coverage (2014-2016), compared with MEX data, MAVEN data were insufficient to allow analysis of the influence of solar wind pressure variation on correlation length.

\subsubsection{Temporal scale}

Figure 4 shows the correlation length in temporal scales for electron density (Figure 4a) and magnetic field (Figure 4b). The mean plasma boundaries are also shown. In the electron density map, the bow shock can be seen clearly in the $C L_{T}$ distribution. The dominant value of $C L$ Tin the magnetosphere of Mars is around 12 



Figure 2. (a) Maps of correlation length around Mars in temporal scale for all MEX data; (b) Maps of correlation length during periods of low solar wind pressure ( $<1 \mathrm{nPa})$; and (c) Maps of correlation length during periods of high solar wind pressure $(1-100 \mathrm{nPa})$.

seconds, but as was seen in the MEX analysis, the region of the subsolar point is dominated by high values of $C L_{T}$ in the solar wind (11-15 seconds), bow shock, dayside of the magnetosheath (16 seconds), and MPR (13-15 seconds). In Figure $4 \mathrm{~b}$ it is possible to notice that the $C L_{T}$ is slightly lower in the magnetic field than in the electron density data (Figure 4a). This difference may be caused by different factors: First, the SWEA density measurements depend strongly on spacecraft attitude and solar illumina- 

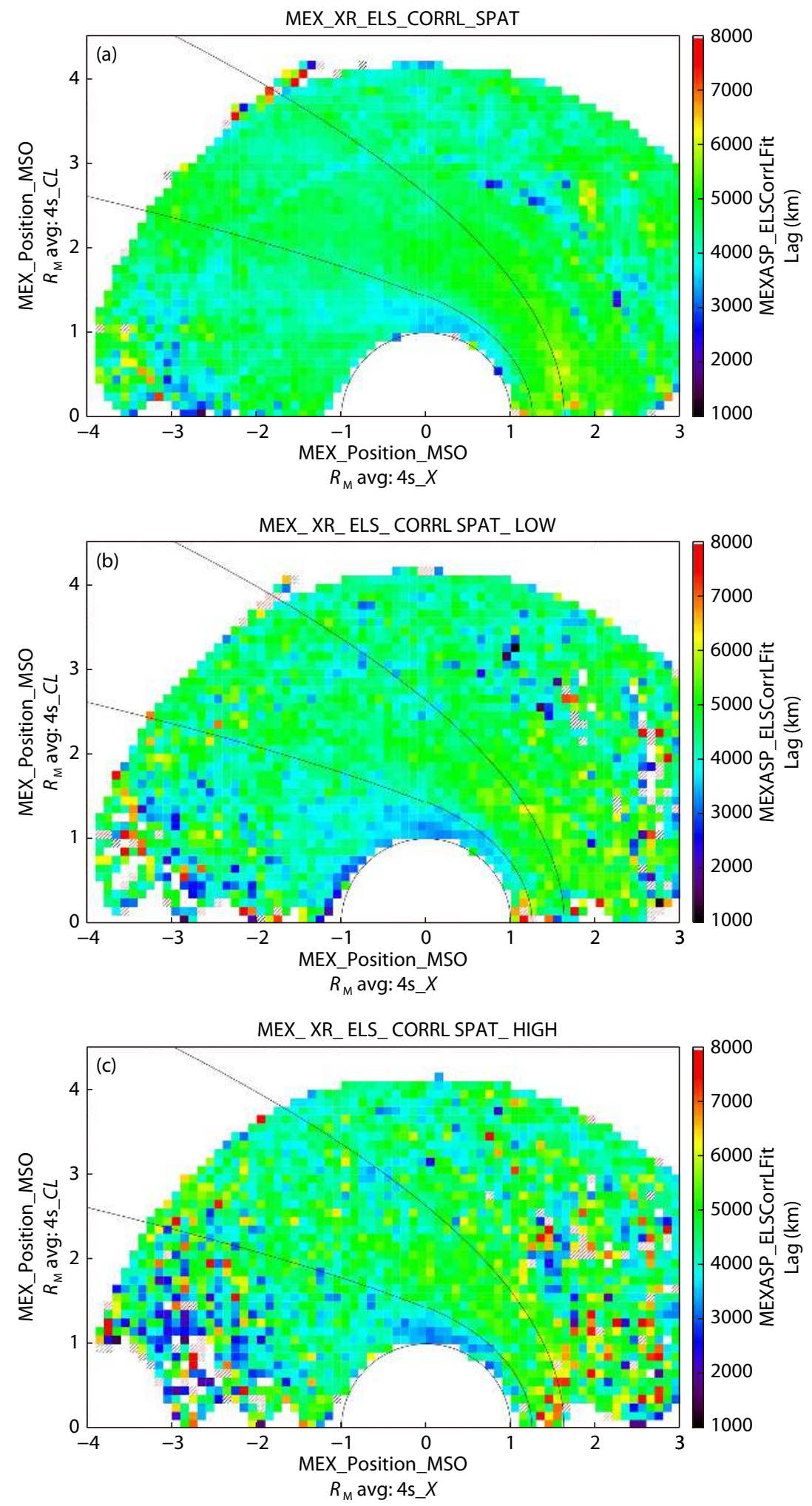

Figure 3. (a) Maps of the correlation length of ULF waves around Mars in spatial scale for all MEX data; (b) Maps of correlation length during periods of low solar wind pressure ( $<1 \mathrm{nPa})$; and (c) Maps of correlation length during periods of high solar wind pressure (1-100 $\mathrm{nPa})$.

tion (via spacecraft potential), which can affect our results. Second, a physical explanation would be that there are more rotational Alfven waves in the solar wind plasma that are not visible in the total magnetic field (where only transversal Alfvenic waves can be detected), and the rotational waves may affect the elec- tron distribution. In Figure 4b, the region that has a larger $C L_{T}$ domain is the magnetosheath, where $C L_{T} S$ are about 13-15 seconds. The bow shock cannot be as clearly observed here as it was in the electron density analysis; here we calculate its average $C L_{T}$ as about 12 seconds. In the MPR, the $C L_{T}$ is around 8 seconds, the 

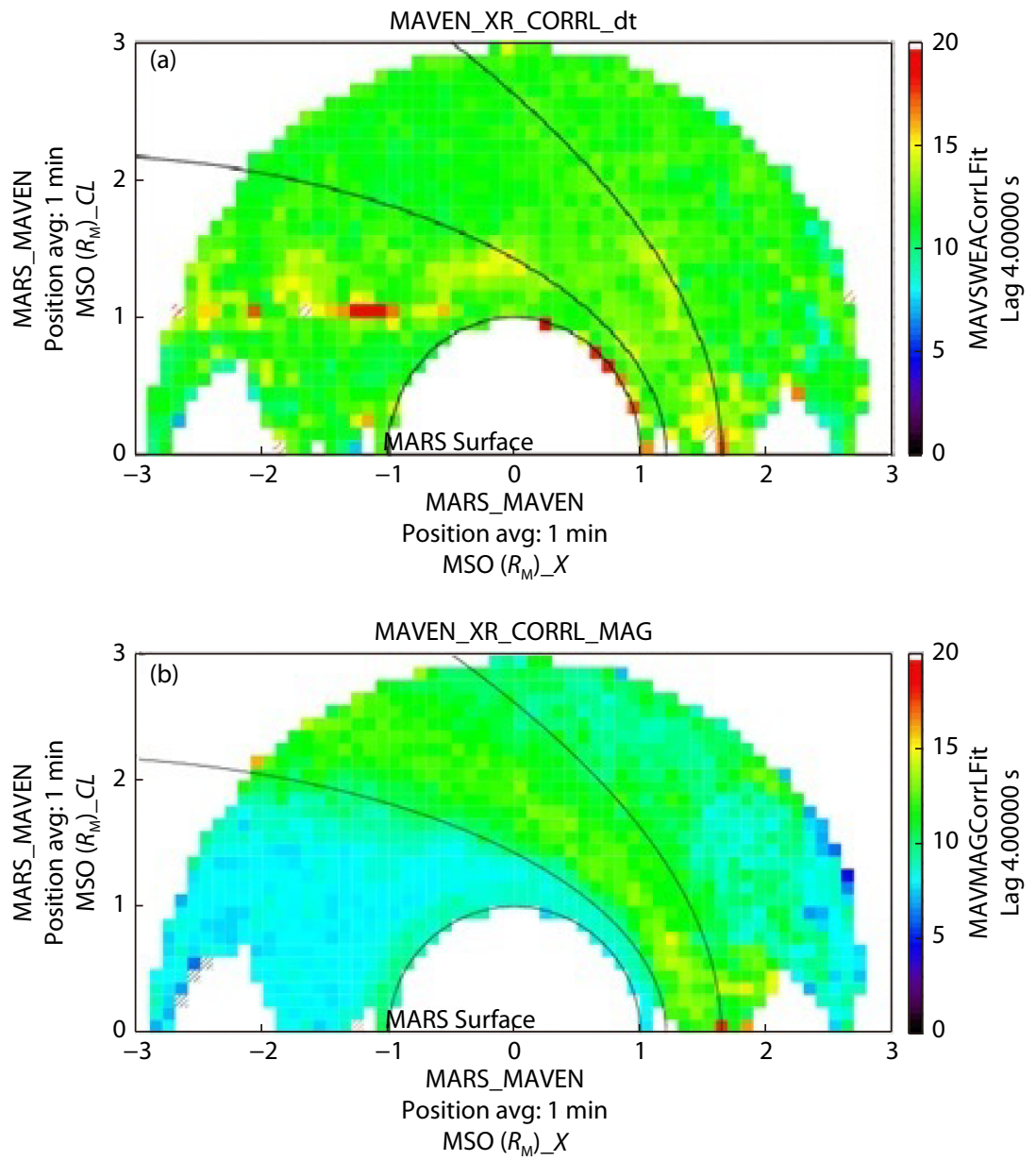

Figure 4. (a) Maps of the correlation length of ULF waves around Mars using MAVEN electron density data in temporal scale; (b) Maps of correlation length around Mars using MAVEN magnetic field data in temporal scale.

same value as observed at the tail. Taking into account that we here use $4 \mathrm{~s}$ time resolution data this means we cannot detect in these data a significant $C L_{T}$ lower than 8 seconds.

\subsubsection{Spatial scale}

The $C L_{S}$ in spatial scale using MAVEN data maps for electron density and magnetic field are shown in Figures $5 \mathrm{a}$ and $5 \mathrm{~b}$, respectively. Here, the color scale representing the $C L_{s}$ in kilometers varies from 0 to $1.0 \times 10^{4} \mathrm{~km}$. In Figure $5 \mathrm{a}$, larger $C L_{s}$ is observed in the solar wind. The magnetosheath shows low values of correlation length for the most part of the dayside and flank $\left(2.5 \times 10^{3}\right.$ $\mathrm{km})$, but a correlation length of around $4.5 \times 10^{3}-5.0 \times 10^{3} \mathrm{~km}$ can be observed in the subsolar point.

A map similar to that of the electron density data is observed in the spatial analysis of $C L_{s}$ derived from the magnetic field data (Figure 5b), but again, we observe smaller scales. The characteristic $C L_{s}$ in solar wind here varies between $4.0 \times 10^{3}-5.0 \times 10^{3} \mathrm{~km}$. Similar values are observed at the nightside of the magnetosheath and bow shock. At the dayside, the dominant $C L_{s}$ in the magnetosheath is around $2.0 \times 10^{3} \mathrm{~km}$. The MPR exhibits $C L_{s}$ of about $1.5 \times 10^{3} \mathrm{~km}$; in the tail, where the smallest correlation length is observed, $C L_{S}$ varies between $1.5 \times 10^{3}$ and $2.0 \times 10^{3} \mathrm{~km}$.

\section{Discussion}

Comparing the values of $C L_{T}$ found from MEX data in temporal scale (Figure 2a) with what was found for MAVEN data (Figure 4a), it is possible to notice that the $C L_{T} S$ are similar in both analyses. In the spatial analysis, $C L_{s} s$ are on the same scale, but the MEX data yield slightly larger values (Figure 3a). This difference is probably a consequence of the fact that the $C L_{S}$ s in spatial scale are computed using the solar wind velocity, a parameter whose large fluctuations can influence the results. That effect may be more prominent in the MEX results, which were based on a significantly longer interval of time (April 2004-2015) with more variation in the solar wind speed for that spacecraft, as compared with the MAVEN interval (November 2014-2016).

The $C L_{s}$ values obtained in spatial scale for electron density (MEX and MAVEN) and magnetic field (MAVEN) in the magnetosheath and MPR were compared with the mean size of these regions (Table 1).

The size of the plasma regions was obtained from the difference between the location of the plasma boundaries; bow shock (Vignes et al., 2002); MPB (Nagy et al., 2004); and ionopause (Trotignon et al., 2006). The $C L_{s}$ and the ratio between them are also presented. For the MEX electron density analysis, $C L_{s}$ at the dayside of the magnetosheath is approximately five times the 

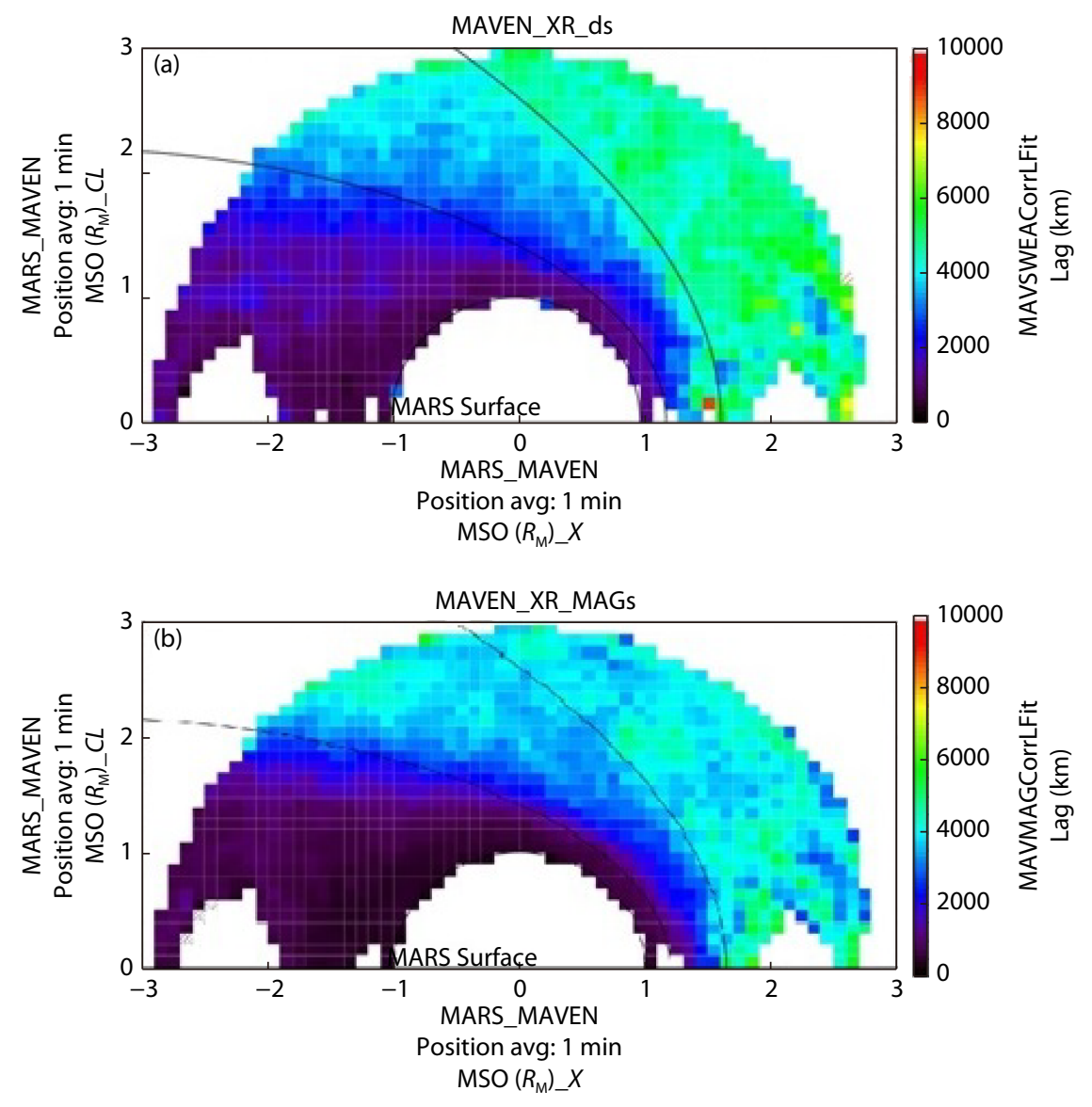

Figure 5. (a) Maps of the correlation length of ULF waves around Mars using MAVEN electron density data in spatial scale; (b) Maps of correlation length around Mars using magnetic field MAVEN data in spatial scale.

magnetosheath size, whereas $C L$ at the MPB/MPR is 25 times larger than that region. Although the ratio between $C L_{s}$ and plasma region is smaller in the MAVEN analysis than the ratio obtained for MEX (explained above as probably attributable to high fluctuations in solar wind velocity), it was seen that in the MAVEN analyses $C L_{S} s$ are still larger than the plasma regions, even in the magnetic field data. This result can be considered as another indication that wave fluctuations at the magnetosheath and MPR are correlated with oscillations at the ionosphere, caused by ULF waves that can penetrate into the ionopause. We interpret this as oscillations from the magnetosheath causing pressure pulses at the ionopause, and as a response to these, the ionospheric plasma pushes back the boundary. Thus the energy from these waves is transferred to the ionospheric plasma, accelerating planetary ions away from the planet.

\section{Conclusions}

Correlation lengths in the plasma environments of Mars have been identified here for the first time, using data of two spacecraft, MEX (2005-2015) and MAVEN (2014-2016). The influence of solar wind pressure variation was also analyzed. In temporal scale, the correlation length in electron density data in the plasma boundaries and regions of the Martian magnetosphere varies between 13 and 17 seconds in the MEX analysis and between 11 and 16 seconds in the MAVEN analysis. In the magnetic field data (MAVEN) it varies between 8-15 seconds. In spatial scale the dominant correlation length is around $5.5 \times 10^{3} \mathrm{~km}$ for MEX electron

Table 1. Comparison of the highest correlation length in the plasma region of the magnetosphere of Mars with the size of them

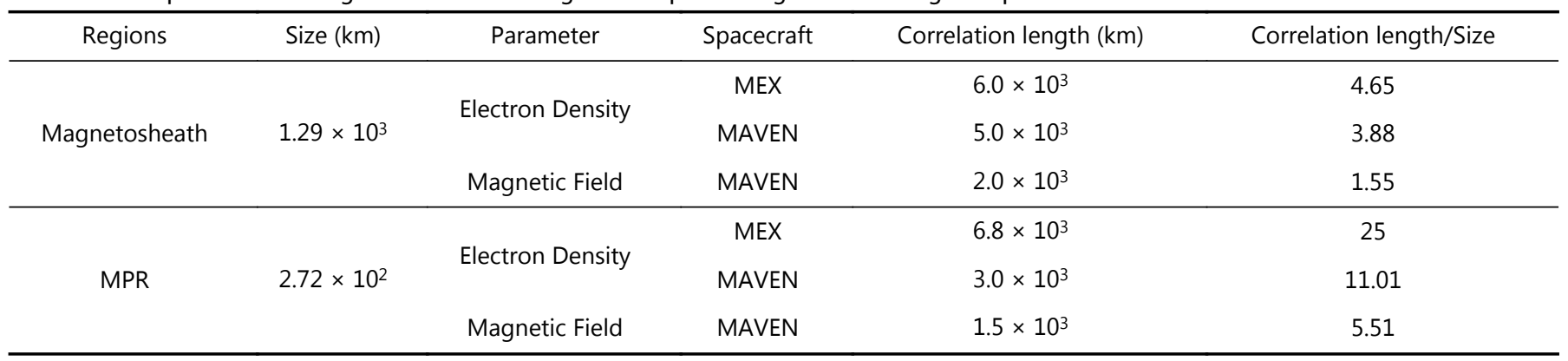


density data. In the MAVEN analysis, correlation length of $4.5 \times$ $10^{3} \mathrm{~km}$ is observed at the dayside of the magnetosheath; lower values are observed in the inner magnetosphere $\left(2 \times 10^{3}-3 \times\right.$ $10^{3} \mathrm{~km}$ ). In the magnetic field analysis, the $C L_{s}$ varies between $1 \times$ $10^{3}$ and $5 \times 10^{3} \mathrm{~km}$. The solar wind pressure shows a significant influence on the correlation length values, which are higher during periods of high solar wind pressure.

Large correlation lengths were observed at the nose of the planet; those values can be seen throughout the magnetosheath until the inner magnetosphere. These observations indicate that waves at the magnetosheath can be related to oscillations in the ionosphere. This means that, in a local region, wave trains can cause resonance effects at the planetary ionopause, which consequently may contribute to enhanced ion escape from the Martian atmosphere. This conclusion is reaffirmed by the tabular comparison between region size and regional correlation lengths in the dayside plasma regions of Mars, where correlation lengths for both electron density data (MEX and MAVEN) and magnetic field data (MAVEN) are larger than the lengths of the regions. This indicates that wave fluctuations at the magnetosheath and MPR may be correlated with oscillations in the upper ionosphere, due to ULF waves that can cause a pressure resonance at the ionopause. This can be an important mechanism to extract ions from Mars and may have influenced the long-term evolution of its atmosphere.

\section{Acknowledgments and Data}

We would like to thank the Mars Express ASPERA team and also MAVEN SWEA and MAG teams for providing excellent data quality. AMSF would like to thank FAPESP agency for support (projects 2016/10794-2 and 2017/00516-8). The MJAB was supported by FAPEG (grant no. 201210267000905) and CNPq (grants no. 302330/2015-1). EE thanks CNPq (project CNPq/PQ 302583/20157) and FAPESP (2018/21657-1) agencies for their support.

\section{References}

Archer, M., Horbury, T. S., Lucek, E. A., Mazelle, C., Balogh, A., and Dandouras, I. (2005). Size and shape of ULF waves in the terrestrial foreshock. J. Geophys. Res. Space Phys., 110(A5), A05208. https://doi.org/10.1029/2004JA010791

Barabash, S., Lundin, R., Andersson, H., Gimholt, J., Holmström, M., Noberg, O., Yamauchi, M., Asamura, K., Coates, A. J., ... Bochsler, P. (2004). ASPERA-3: Analyser of space plasmas and energetic ions for Mars Express. In A. Wilson (Ed.), Mars Express: the Scientific Payload (pp. 121-139). Noordwijk, Netherlands: ESA Publications Division.

Cloutier, P. A., and Daniell, Jr. R. E. (1973). Ionospheric currents induced by solar wind interaction with planetary atmospheres. Planet. Space Sci., 21(3), 463-474. https://doi.org/10.1016/0032-0633(73)90043-3

Cloutier, P. A., and Daniell, Jr. R. E. (1979). An electrodynamic model of the solar wind interaction with the ionospheres of Mars and Venus. Planet. Space Sci., 27(8), 1111-1121. https://doi.org/10.1016/0032-0633(79)90082-5

Collinson, G., Wilson III, L. B., Omidi, N., Sibeck, D., Espley, J., Fowler, C. M., Mitchell, D., Grebowsky, J., Mazelle, C., ... Jakosky, B. (2018). Solar wind induced waves in the skies of Mars: lonospheric compression, energization, and escape resulting from the impact of ultralow frequency magnetosonic waves generated upstream of the Martian bow shock. J. Geophys. Res. Space Phys., 123(9), 7241-7256. https://doi.org/10.1029/2018JA025414

Connerney, J. E. P., Espley, J., Lawton, P., Murphy, S., Odom, J., Oliversen, R., and Sheppard, D. (2015a). The MAVEN magnetic field investigation. Space Sci. Rev., 195(1-4), 257-291. https://doi.org/10.1007/s11214-015-0169-4
Connerney, J. E. P., Espley, J. R., DiBraccio, G. A., Gruesbeck, J. R., Oliversen, R. J., Mitchell, D. L., Halekas, J., Mazelle, C., Brain, D., and Jakosky, B. M. (2015b). First results of the MAVEN magnetic field investigation. Geophys. Res. Lett., 42(21), 8819-8827. https://doi.org/10.1002/2015GL065366

Delva, M., and Dubinin, E. (1998). Upstream ULF fluctuations near Mars. J. Geophys. Res. Space Phys., 103(A1), 317-326.

https://doi.org/10.1029/97JA02501

Espley, J. R., Cloutier, P. A., Brain, D. A., Crider, D. H., and Acuña, M. H. (2004). Observations of low-frequency magnetic oscillations in the Martian magnetosheath, magnetic pileup region, and tail. J. Geophys. Res. Space Phys., 109(A7), A07213. https://doi.org/10.1029/2003JA010193

Fisk, L. A., and Sari, J. W. (1973). Correlation length for interplanetary magnetic field fluctuations. J. Geophys. Res., 78(28), 6729-6736. https://doi.org/10.1029/JA078i028p06729

Fränz, M., Dubinin, E., Roussos, E., Woch, J., Winningham, J. D., Frahm, R., Coates, A. J., Fedorov, A., Barabash, S., and Lundin, R. (2006). Plasma moments in the environment of Mars. Mars express ASPERA-3 observations. Space Sci. Rev., 126(1-4), 165-207. https://doi.org/10.1007/s11214-006-9115-9

Fränz, M., Echer, E., Marques De Souza, A., Dubinin, E., and Zhang, T. L. (2017). Ultra low frequency waves at Venus: observations by the Venus express spacecraft. Planet. Space Sci., 146, 55-65. https://doi.org/10.1016/j.pss.2017.08.011

Halekas, J. S., Ruhunusiri, S., Harada, Y., Collinson, G., Mitchell, D. L., Mazelle, C., Mcfadden, J. P., Connerney, J. E. P., Espley, J. R., ... Jakosky, B. M. (2017). Structure, dynamics, and seasonal variability of the Mars-solar wind interaction: MAVEN Solar Wind lon Analyzer in-flight performance and science results. J. Geophys. Res. Space Phys., 122(1), 547-578. https://doi.org/10.1002/2016JA023167

Han, X., Fräenz, M., Dubinin, E., Wei, Y., Andrews, D. J., Wan, W., He, M., Rong, Z. J., Chai, L., ... Barabash, S. (2014). Discrepancy between ionopause and photoelectron boundary determined from Mars Express measurements. Geophys. Res. Lett., 41(23), 8221-8227. https://doi.org/10.1002/2014GL062287

Kivelson, M. G. (1995). Pulsations and magnetohydrodynamic waves. In M. G. Kivelson, et al. (Eds.), Introduction to Space Physics (pp. 1875-1883). Cambridge: Cambridge University Press.

Kivelson, M. G., and Bagenal, F. (2007). Planetary magnetospheres. In P. R. Weissman, et al. (Eds.), Encyclopedia of the Solar System (pp. 519-539). San Diego, CA: Academic.

Luhmann, J. G., Russell, C. T., Brace, L. H., and Vaisberg, O. L. (1992). The intrinsic magnetic field and solar wind interaction of Mars. In H. Kieffer, et al. (Eds.), Mars (pp. 1090-1134). Tucson, Arizona: University of Arizona Press.

Luhmann, J. G., Ledvina, S. A., and Russell, C. T. (2004). Induced magnetospheres. Adv. Space Res., 33(11), 1905-1912. https://doi.org/10.1016/j.asr.2003.03.031

Lundin, R., Winningham, D., Barabash, S., Frahm, R. A., Andersson, H., Holmström, M., Grigoriev, A., Yamauchi, M., Borg, H., ... Bochsler, P. (2006). Ionospheric plasma acceleration at Mars: ASPERA-3 results. Icarus, 182(2), 308-319. https://doi.org/10.1016/j.icarus.2005.10.035

Lundin, R., Barabash, S., Fedorov, A., Holmstrom, M., Nilsson, H., Sauvaud, J. A., and Yamauchi, M. (2008). Solar forcing and planetary ion escape from Mars. Geophys. Res. Lett., 35(9), L09203. https://doi.org/10.1029/2007GL032884

Lundin, R., Barabash, S., Dubinin, E., Winningham, D., and Yamauchi, M. (2011). Low-altitude acceleration of ionospheric ions at Mars. Geophys. Res. Lett., 38(8), L08108. https://doi.org/10.1029/2011GL047064

Mela, K., and Louie, J. N. (2001). Correlation length and fractal dimension interpretation from seismic data using variograms and power spectra. Geophysics, 66(5), 1372-1378. https://doi.org/10.1190/1.1487083

Mitchell, D. L., Mazelle, C., Sauvaud, J. A., Thocaven, J. J., Rouzaud, J., Fedorov, A., Rouger, P., Toublanc, D., Taylor, E., ... Jakosky, B. M. (2016). The MAVEN solar wind electron analyzer. Space Sci. Rev., 200(1-4), 495-528. https://doi.org/10.1007/s11214-015-0232-1

Morales, J. J., and Nuevo, M. J. (1993). Physical meaning of the time-correlation length obtained in a computer simulation. Phys. Rev. E, 48(2), 1550-1553. https://doi.org/10.1103/physreve.48.1550 
Nagy, A. F., Winterhalter, D., Sauer, K., Cravens, T. E., Brecht, S., Mazelle, C., Crider, D., Kallio, E., Zakharov, E., ... Trotignon, J. G. (2004). The plasma environment of Mars. Space Sci. Rev., 111(1-2), 33-114. https://doi.org/10.1023/B:SPAC.0000032718.47512.92

Palumbo, D. (2012). Determining correlation and coherence lengths in turbulent boundary layer flight data. J. Sound Vib., 331(16), 3721-3737. https://doi.org/10.1016/j.jsv.2012.03.015

Parhi, S., Bieber, J. W., Matthaeus, W. H., and Burger, R. A. (2002). Sensitivity of cosmic ray modulation to the correlation length. Geophys. Res. Lett., 29(8), 99-1-99-3. https://doi.org/10.1029/2001GL013893

Podgorny, I. M., Dubinin, E. M., and Israelevich, P. L. (1980). Laboratory simulation of the induced magnetospheres of comets and Venus. Moon Planets, 23(3), 323-338. https://doi.org/10.1007/BF00902047

Ramstad, R., Barabash, S., Futaana, Y., and Holmström, M. (2017). Solar windand EUV-dependent models for the shapes of the Martian plasma boundaries based on Mars Express measurements. J. Geophys. Res. Space Phys., 122(7), 7279-7290. https://doi.org/10.1002/2017JA024098

Ruhunusiri, S., Halekas, J. S., Connerney, J. E. P., Espley, J. R., McFadden, J. P., Larson, D. E., Mitchell, D. L., Mazelle, C., and Jakosky, B. M. (2015a). Lowfrequency waves in the Martian magnetosphere and their response to upstream solar wind driving conditions. Geophys. Res. Lett., 42(21), 8917-8924. https://doi.org/10.1002/2015GL064968

Ruhunusiri, S., Halekas, J. S., Connerney, J. E. P., Espley, J., Larson, D., and Mitchell, D. L. (2015b). MAVEN characterization of low-frequency plasma waves in the Martian magnetosphere. In Proceedings of the 46th Lunar and Planetary Science Conference. Woodlands, Texas: LPI Contribution.

Russell, C. T., Luhmann, J. G., Schwingenschuh, K., Riedler, W., and Yeroshenko, Y. (1990). Upstream waves at Mars: Phobos observations. Geophys. Res. Lett.,
17(6), 897-900. https://doi.org/10.1029/GL017i006p00897

Schwingeschuh, K., Riedler, W., Zhang, T. L., Lichtenegger, H., Rosenbauer, H., Livi, S., Gevai, G., Gringauz, K., Verigin, M., ... Luhmann, J. G. (1992). The Martian magnetic field environment: induced or dominated by an intrinsic magnetic field? . Adv. Space Res., 12(9), 213-219.

https://doi.org/10.1016/0273-1177(92)90333-S

Trotignon, J. G., Grard, R., and Skalsky, A. (1993). Position and shape of the Martian bow shock: the Phobos 2 plasma wave system observations. Planet. Space Sci., 41(3), 189-198. https://doi.org/10.1016/0032-0633(93)90058-A

Trotignon, J. G., Mazelle, C., Bertucci, C., and Acuña, M. H. (2006). Martian shock and magnetic pile-up boundary positions and shapes determined from the Phobos 2 and Mars global surveyor data sets. Planet. Space Sci., 54(4), 357-369. https://doi.org/10.1016/j.pss.2006.01.003

Vignes, D., Acuña, M. H., Connerney, J. E. P., Crider, D. H., Rème, H., and Mazelle, C. (2002). Factors controlling the location of the bow shock at Mars. Geophys. Res. Lett., 29(9), 42-1. https://doi.org/10.1029/2001GL014513

Volwerk, M., Zhang, T. L., Delva, M., Vörös, Z., Baumjohann, W., and Glassmeier, K. H. (2008). First identification of mirror mode waves in Venus' Magnetosheath?. Geophys. Res. Lett., 35(12), L12204. https://doi.org/10.1029/2008GL033621

Wicks, R. T., Owens, M. J., and Horbury, T. S. (2010). The variation of solar wind correlation lengths over three solar cycles. Solar Phys., 262(1), 191-198. https://doi.org/10.1007/s11207-010-9509-4

Winningham, J. D., Frahm, R. A., Sharber, J. R., Coates, A. J., Linder, D. R., Soobiah, Y., Kallio, E., Espley, J. R., Lundin, R., ... Dierker, C. (2006). Electron oscillations in the induced martian magnetosphere. Icarus, 182(2), 360-370. https://doi.org/10.1016/j.icarus.2005.10.033 